\title{
Crossing Line Profile: A New Approach to Detecting Defects in Aluminium Die Casting
}

\author{
Domingo Mery \\ Departamento de Ingeniería Informática \\ Universidad de Santiago de Chile \\ Av. Ecuador 3659, Santiago de Chile \\ dmery@ieee.org \\ http://www.diinf.usach.cl/ dmery
}

\begin{abstract}
Radioscopy is the accepted way for controlling the quality of aluminium die cast pieces through computer-aided analysis of X-ray images. Two classes of regions are possible in a digital X-ray image of a casting: regions belonging to regular structures of the specimen, and those relating to defects. Since the contrast between a flaw and a defectfree neighbourhood is distinctive, the detection is usually performed by thresholding this feature. Nevertheless, this measurement suffers from accuracy error when the neighbourhood is not homogeneous, for example when the flaw is at an edge of a regular structure of the test object. For this reason, many approaches use a-priori information about the location of regular structures of the test piece. In this paper, a new approach to detecting defects without a-priori knowledge is proposed. The approach is based on features extracted from crossing line profiles, i.e., the grey level profiles along straight lines crossing each segmented potential flaw in the middle. The profile that contains the most similar grey levels in the extremes is selected. Hence, the homogeneity of the neighbourhood is ensured. Features from the selected profile are extracted. The detection performance of our features and a vast number of other known features are assessed by computing the area $A_{z}$ under the Receiver Operation Characteristic (ROC) curve. The best performance is achieved using one of the proposed features yielding an area $A_{z}=0.9944$ in 50 X-ray images of aluminium wheels with 23.000 potential flaws.
\end{abstract}

Keywords: flaw detection, automated visual inspection, X-ray testing.

\section{Introduction}

Shrinkage as molten metal cools during the manufacture of die castings, can cause defect regions within the work piece. These are manifested, for example, by bubble-shaped voids, cracks, slag formations or inclusions (see an example in Fig. 1a). Light-alloy castings produced for the automotive industry, such as wheel rims, steering knuckles and steering gear boxes are considered important components for overall roadworthiness. To ensure the safety of construction, it is necessary to check every part thoroughly. Radioscopy rapidly became the accepted way for controlling the quality of die cast pieces through computer-aided 
analysis of X-ray images [1]. The purpose of this non-destructive testing (NDT) method is to identify casting defects, which may be located within the piece and thus are undetectable to the naked eye. The automated visual inspection of castings is a quality control task to determine automatically whether a casting complies with a given set of product and product safety specifications.

Two classes of regions are possible in a digital X-ray image of an aluminium casting: regions belonging to regular structures of the specimen, and those relating to defects. The automatic process used in fault detection in aluminium castings consists of five steps: a) Image formation, in which an X-ray image of the casting under test is taken and stored in the computer. b) Image pre-processing, where the quality of the X-ray image is improved in order to enhance the details of the X-ray image. c) Image segmentation, in which each potential flaw of the $\mathrm{X}$-ray image is found and isolated from the rest of the scene. d) Feature extraction, where the potential flaws are measured and some significant characteristics are quantified. e) Classification, where the extracted features of each potential flaw are analysed and assigned to one of the classes (regular structure or defect).

In an X-ray image we can see that the defects, such as voids, cracks and bubbles (or inclusions and slags), show up as bright (or dark) features. The reason is that the X-ray attenuation in these areas is shorter (or higher). Since the contrast in the X-ray image between a flaw and a defect-free neighbourhood of the specimen is distinctive, the detection is usually performed by analysing this feature. There are several definitions of contrast, they generally give a comparison between the grey level of a region (potential flaw) and the grey level of its corresponding neighbourhood (see for example [2]). Nevertheless, the last measurement suffers from accuracy error when the neighbourhood is not ho-
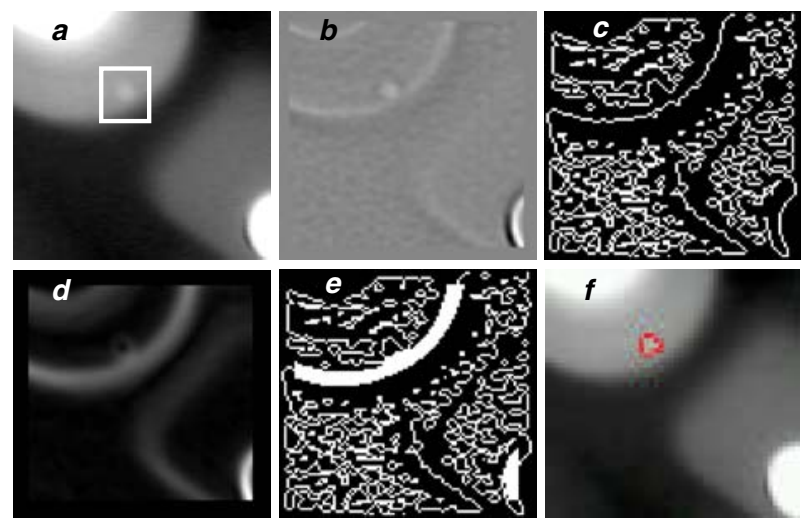

Fig. 1. Detection of flaws: a) radioscopic image with a small flaw at an edge of a regular structure, b) Laplacian-filtered image with $\sigma=1.25$ pixels (kernel size $=11 \times 11$ ), c) zero crossing image, d) gradient image, e) edge detection after adding high gradient pixels, and f) detected flaw using feature $F_{1}$ extracted from a crossing line profile. 
mogeneous, for example when the flaw is at an edge of a regular structure of the test object. For this reason, many approaches compute the grey level of the neighbourhood using a-priori knowledge of the design structure of the test piece (see for example $[3,4]$ ). Thus, the defect-free areas are defined in sections where the grey level values have a small variance. These methods have become the most widely established in industrial applications owing to their high detection performance $^{1}$. However, they require a very precise positioning of the test object and a complicated selection process of the defect-free areas. A review of the existing approaches of automated flaw detections in aluminium castings can be found in [1].

In order to avoid the mentioned problems, this paper proposes a new approach based on features extracted from crossing line profiles computed without a-priori knowledge. The paper is organised as follows. In Section 2, the segmentation of potential flaws is outlined. The new approach is described in Section 3. The performance analysis is presented in Section 4. Finally, Section 5 gives concluding remarks. An early version of this research is presented in [2].

\section{Segmentation of potential flaws}

The X-ray image taken with an image intensifier and a CCD camera (or a flat panel detector), must be pre-processed to improve the quality of the image. In our approach, the pre-processing techniques are used to remove noise, enhance contrast, correct the shading effect and restore blur deformation [1].

The segmentation of potential flaws identifies regions in radioscopic images that may correspond to real defects. Two general characteristics of the defects are used to identify them: a) a flaw can be considered as a connected subset of the image, and b) the grey level difference between a flaw and its neighbourhood is significant. According to the mentioned characteristics, a simple automated segmentation approach was suggested in [5] (see Fig. 1). First, a Laplacianof-Gaussian (LoG) kernel and a zero crossing algorithm [6] are used to detect the edges of the X-ray images. The LoG-operator involves a Gaussian lowpass filter which is a good choice for the pre-smoothing of our noisy images that are obtained without frame averaging. The resulting binary edge image should produce at real flaws closed and connected contours which demarcate regions. However, a flaw may not be perfectly enclosed if it is located at an edge of a regular structure as shown in Fig. 1c. In order to complete the remaining edges of these flaws, a thickening of the edges of the regular structure is performed as follows: a) the gradient of the original image is calculated (see Fig. 1d); b) by thresholding the gradient image at a high grey level a new binary image is obtained; and c) the resulting image is added to the zero crossing image (see Fig. 1e). Afterwards, each closed region is segmented as potential flaw. For details see a description of the method in [5].

\footnotetext{
${ }^{1}$ By inspecting the pieces used in this work, the results obtained by an industrial software based on a-priori knowledge were excellent: $100 \%$ of the real flaws were detected without false alarms [5].
} 
This is a very simple detector of potential flaws with a large number of false alarms flagged erroneously. However, the advantages are as follows: a) it is a single detector (it is the same detector for each image), b) it is able to identify potential defects independent of the placement and the structure of the specimen, i.e., without a-priori information of the design structure of the test piece, and c) the detection rate of real flaws is very high (approximately 90\%). In order to reduce the number of the false positives, the segmented regions must be measured and classified. In the next Section, we present new features that can be used to classify with high accuracy the segmented regions in one of the two classes: regular structure or defect.

\section{Crossing line profiles}

As explained in Section 2, a segmented potential flaw is defined as a region enclosed by edges of the binary image obtained in the edge detection (see connected black pixels in Fig. 1e). For each segmented region, a window $g$ is defined from the X-ray image $x$ as: $g(i, j)=x\left(i+i_{r}, j+j_{r}\right)$ for $i=1, \ldots, 2 h+1$, and $j=1, \ldots, 2 w+1$, where $h$ and $w$ are the height and width of the region. The offsets $i_{r}$ and $j_{r}$ are defined as $i_{r}=i_{0}-h-1$ and $j_{r}=j_{0}-w-1$ where $\left(i_{0}, j_{0}\right)$ denotes the coordinates of the centre of gravity of the region (rounded to the nearest integers). Hence, $g$ is a window of size $(2 h+1) \times(2 w+1)$, in which the middle pixel corresponds to the centre of gravity of the segmented potential flaw, i.e., $g(h+1, w+1)=x\left(i_{0}, j_{0}\right)$.

Now, we define the crossing line profile $P_{\theta}$ as the grey level function along a straight line of window $g$ through the middle pixel $(h+1, w+1)$ forming an angle $\theta$ with $i$-axis. In [2], $P_{0}$ and $P_{\pi / 2}$ were analysed together in order to obtain two features, $K$ and $K_{\sigma}$, that give a measurement of the difference between maximum and minimum, and the standard deviation of both crossing line profiles. However, the analysis does not take into account that the profiles could include a nonhomogeneous area. For example, if a non-flaw region is segmented at an edge of a regular structure, it could be that $P_{0}\left(\right.$ or $P_{\pi / 2}$ ) includes a significant grey level change of the regular structure. In this case, the variation of the profile will be large and therefore the region will be erroneously classified as defect.

In order to solve this problem, we suggest to analyse individually eight crossing line profiles $P_{\theta}$, at $\theta=k \pi / 8$, for $k=0, \ldots, 7$, as illustrated in Fig. 2. In this analysis, the crossing line profile that contains the most similar grey levels in the extremes is selected. Hence, the attempt is made to ensure the homogeneity of the neighbourhood filtering out those profiles that present a high grey level change in the edge of the regular structure. In the example of Fig. 2, the selected profile is obtained for $k=5$ where the grey values of the extremes are both approximately equal to 150 . We observe that the selected crossing line is approximately perpendicular to the direction of the gradient of the X-ray image without defect. This coincides with one of the criteria used by approaches with a-priori knowledge: the selected pixels of the defect-free area are located perpendicular to the direction of the gradient of the piece's contour [1]. 

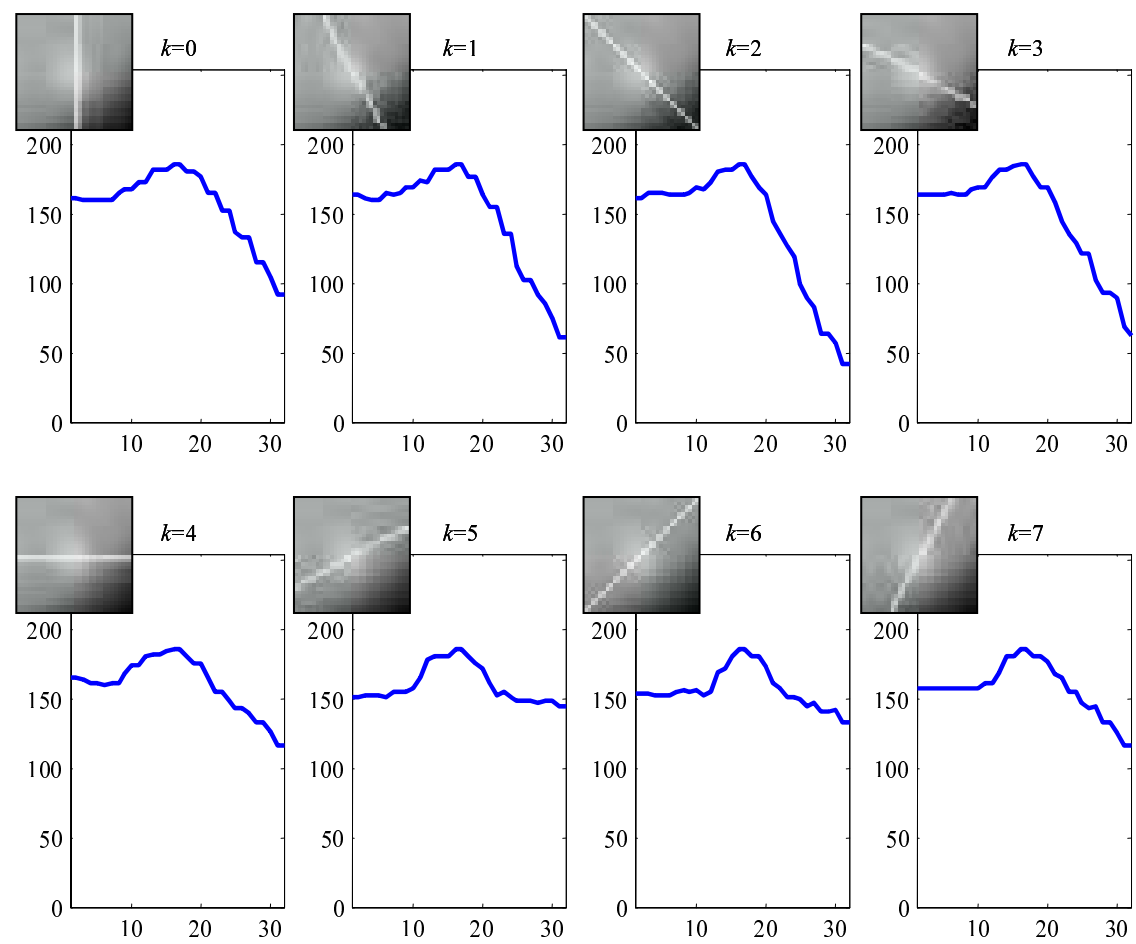

Fig. 2. Crossing line profiles for the window shown in Fig. 1a.

Before the features are extracted, a pre-processing of the selected crossing line profile is performed as follows: 1) The selected profile is resized to size $n=32$ using a nearest neighbour interpolation. The resized profile will be denoted by $P$. 2) In order to obtain a defect profile without the background of the regular structure, $P$ is linearly transformed by $Q_{i}=m P_{i}+b$, for $i=1, \ldots, n$, where $m$ and $b$ are so chosen that $Q_{1}=Q_{n}=0$.

Finally, the proposed features are extracted from the normalised profile $Q$. They are defined as follows: $\bar{Q}$ : mean of $Q ; \sigma_{Q}$ : standard deviation of $Q ; \Delta_{Q}$ : difference between maximum and minimum of $Q$; and $F_{i}$ : magnitude of the $i$-th harmonic of the Discrete Fourier Transform of $Q$ for $i=1, . .4$.

\section{Performance Analysis}

First, we will mention the features that are extracted and analysed in this work. The features are divided into two groups: geometric and gray value features. Although most of the extracted features do not give any information about the contrast, they were analysed in order to investigate if they are relevant to defect detection. 
The geometric features provide information about the size and the shape of the segmented potential flaw. The extracted geometric features are: area, perimeter, height, width, roundness [6], Hu invariant moments, Flusser and Suk invariant moments, Fourier descriptors, and Gupta and Srinath moments [7], semi-minor and semi-major axis of ellipse fitted to the contour of the potential flaw [6], and Danielsson shape factor [8].

The grey value features provide information on the brightness of the segmented potential flaw. In this group, the extracted features are: mean grey value, mean gradient in the boundary, mean second derivate in the region [2], radiographic contrasts, contrasts $K$ and $K_{\sigma}$ based on $P_{0}$ and $P_{\pi / 2}$ [2], invariant moments with grey value information [7], local variance [9], mean and range of the 14 Haralick textural features based on the co-occurrence matrix in four different directions taken neighbouring pixels separated by a distance of $1,2,3$, 4 , and 5 pixels [10], and finally the first 64 components of the discrete Fourier transform, the Karhunen Loève transform and the discrete cosine transform [6] taken from a normalised image window of $32 \times 32$ pixels including potential flaw and neighbourhood.

Each feature is analysed independently using a threshold classifier. Thus, a potential flaw is classified as regular structure if the feature is bellow a threshold, otherwise it will be assigned to the defect class. In order to obtain the feature that yields the best detection performance, the Receiver Operation Characteristic (ROC) [11] curve is analysed, which is a plot of the 'sensitivity' $(S n)$ against the '1-specificity' $(1-S p)$ defined as:

$$
S n=\frac{T P}{T P+F N}, \quad 1-S p=\frac{F P}{T N+F P},
$$

where $T P$ is the number of true positives (flaws correctly classified); $T N$ the number of true negatives (regular structures correctly classified); FP is the number of false positives (false alarms, i.e., regular structures classified as defects); and $F N$ is the number of false negatives (flaws classified as regular structures). Ideally, $S_{n}=1$ and $1-S_{p}=0$, i.e., all flaws are detected without flagging false alarms. The ROC curve permits to assess the detection performance at various operating points (e.g., thresholds in the classification). The area under the ROC curve $\left(A_{z}\right)$ is normally used as performance measure because it indicates how reliable the detection can be performed. A value of $A_{z}=1$ gives perfect classification, whereas $A_{z}=0.5$ corresponds to random guessing.

In our experiments, 50 X-ray images of aluminium wheels were analysed. In the segmentation 22.936 potential flaws were obtained, in which there were 60 real flaws. Some of them were existing blow holes. The other defects were produced by drilling small holes in positions of the casting which were known to be difficult to detect. In the performance analysis, the best result was achieved by our feature $F_{1}$ (see definition in Section 3 ), yielding an area $A_{z}=0.9944$. The ROC curve of this feature and the distribution of samples for the two classes are illustrated in Fig. 3. A detection example is shown in Fig. 1f. The results of the top eight features are summarised in Table 1, in which the areas $A_{z}$ and the specificities obtained at sensitivity levels of $100 \%$ and $95 \%$ are given. 

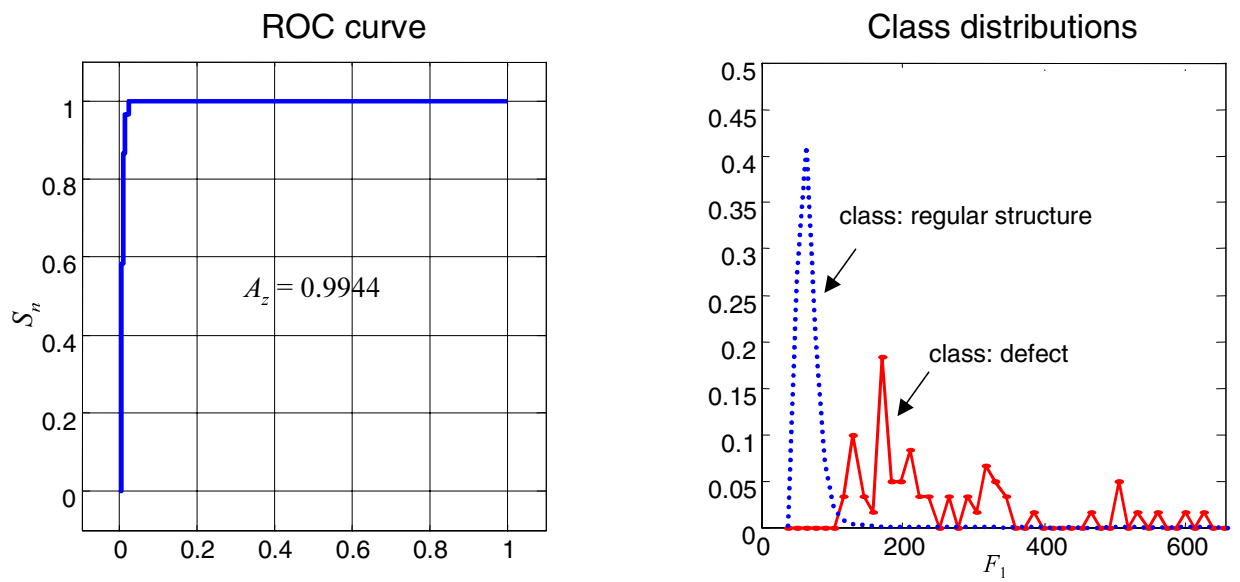

Fig. 3. ROC curve and distribution of classes for the feature $F_{1}$.

Table 1. $A_{z}$ and $S p$ values at $S n=100 \%$ and $95 \%$

\begin{tabular}{|c|c||c|c|c|c|c|c|}
\hline \multicolumn{4}{|c|}{} & \multicolumn{2}{c|}{$@ S_{n}=100 \%(T P=60)$} & $@ S_{n}=95 \%(T P=57)$ \\
\hline Ranking & Feature & Reference & $A_{z}$ & $S p$ & $F P /$ image & $S p$ & $F P /$ image \\
\hline 1 & $F_{1}$ & Section 3 & 0.9944 & 0.9783 & 9.9 & 0.9860 & 6.4 \\
2 & $\sigma_{Q}$ & Section 3 & 0.9927 & 0.9685 & 14.4 & 0.9809 & 8.7 \\
3 & $\Delta_{Q}$ & Section 3 & 0.9901 & 0.9662 & 15.5 & 0.9765 & 10.8 \\
4 & $K s$ & {$[2]$} & 0.9897 & 0.9667 & 15.2 & 0.9727 & 12.5 \\
5 & $K$ & {$[2]$} & 0.9891 & 0.9541 & 21.0 & 0.9639 & 16.5 \\
6 & $\bar{Q}$ & Section 3 & 0.9856 & 0.8835 & 53.3 & 0.9577 & 19.4 \\
7 & $f_{8}(5)$ & {$[10]$} & 0.9725 & 0.6340 & 167.5 & 0.9244 & 34.6 \\
8 & $f_{13}(2)$ & {$[10]$} & 0.9711 & 0.6579 & 156.5 & 0.9037 & 44.1 \\
\hline
\end{tabular}

One of the performances obtained by first feature $\left(S_{p}=0.9860\right.$ at $\left.S_{n}=95 \%\right)$ indicates that on average there are 6.4 false alarms per image when detecting 57 from 60 real flaws. These results are a substantial improvement over those presented in [2], in which, for the same images, the results obtained have a value of $S_{p}=0.9727$ at $S_{n}=95 \%$, which indicates 12.5 false alarms per image when detecting 57 from 60 real flaws. We observe, that the geometric features do not provide relevant information to separate the classes. The reason is because the regions corresponding to flaws and regular structures have similar size and shapes. Furthermore, the top six features are obtained from the crossing line profiles. In addition, there are two textural features with high values of $A_{z}$, $f_{8}(5)$ and $f_{13}(2)$, that correspond to the mean sum entropy by a distance of 5 pixels and mean of information measure of correlation by a distance of 2 pixels respectively. However, the sensitivity of $100 \%$ is achieved at low specificity levels. 


\section{Concluding remarks}

In this paper, a new approach to detecting defects in castings without a-priori knowledge of the design structure is proposed. The approach is based on features extracted from crossing line profiles, i.e., grey level profiles along lines crossing segmented potential flaws in the middle. Several features obtained from the crossing line profiles were suggested. The detection performance of our features and more than 350 other features are assessed by computing the area $A_{z}$ under the ROC curve. The best performance $\left(A_{z}=0.9944\right)$ was achieved using the suggested feature $F_{1}$ calculated as the amplitude of the first harmonic of a normalised crossing line profile. This means, that only 6.4 false alarms per image are obtained in the identification of potential flaws (at $S_{n}=95 \%$ ). By combining more than one non-correlated a better classification can be carried out [12]. It is known that false alarms flagged in this step can be eliminated using a posterior analysis based on image sequence analysis without eliminating the real flaws [5].

\section{References}

1. Mery, D., Filbert, D., Jaeger, T.: Image processing for fault detection in aluminum castings. In MacKenzie, D., Totten, G., eds.: Analytical Characterization of Aluminum and Its Alloys, New York, Marcel Dekker (2003) (In Press).

2. Mery, D., Filbert, D.: Classification of potential defects in automated inspection of aluminium castings using statistical pattern recognition. In: $8^{\text {th }}$ European Conference on Non-Destructive Testing (ECNDT 2002), Barcelona (2002)

3. Filbert, D., Klatte, R., Heinrich, W., Purschke, M.: Computer aided inspection of castings. In: IEEE-IAS Annual Meeting, Atlanta, USA (1987) 1087-1095

4. Schulenburg, H., Purschke, M.: Advances in the automatic evaluation of radioscopic images. In: International Conference on Computerized Tomography for Industrial Applications and Image Processing in Radiology, March 15-17, Berlin (1999) 241243

5. Mery, D., Filbert, D.: Automated flaw detection in aluminum castings based on the tracking of potential defects in a radioscopic image sequence. IEEE Trans. Robotics and Automation 18 (2002) 890-901

6. Castleman, K.: Digital Image Processing. Prentice-Hall, Englewood Cliffs, New Jersey (1996)

7. Sonka, M., Hlavac, V., Boyle, R.: Image Processing, Analysis, and Machine Vision. 2 edn. PWS Publishing, Pacific Grove, CA (1998)

8. Danielsson, P.E.: A new shape factor. Computer Graphics and Image Processing 7 (1978) 292-299

9. Jähne, B.: Digitale Bildverarbeitung. 2 edn. Springer, Berlin, Heidelberg (1995)

10. Haralick, R., Shanmugam, K., Dinstein, I.: Textural features for image classification. IEEE Trans. on Systems, Man, and Cybernetics SMC-3 (1973) 610-621

11. Egan, J.: Signal detection theory and ROC analysis. Academic Press, New York (1975)

12. Mery, D., da Silva, R., Caloba, L., Rebello, J.: Pattern recognition in the automatic inspection of aluminium castings. In: International Conference on Computerized Tomography for Industrial Applications and Image Processing in Radiology, June 23-25, Berlin (2003) (accepted). 